NISTIR 8180

\title{
Method to Improve Point-Based Registration by Restoring Rigid-Body Condition
}

\author{
Marek Franaszek \\ Geraldine S. Cheok
}

This publication is available free of charge from:

https://doi.org/10.6028/NIST.IR.8180

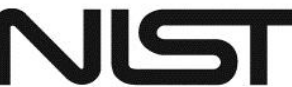

National Institute of Standards and Technology U.S. Department of Commerce 


\title{
Method to Improve Point-Based Registration by Restoring Rigid-Body Condition
}

\author{
Marek Franaszek \\ Geraldine S. Cheok \\ Intelligent Systems Division \\ Engineering Laboratory
}

This publication is available free of charge from: https://doi.org/10.6028/NIST.IR.8180

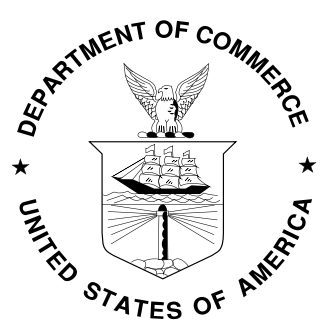

U.S. Department of Commerce Wilbur L. Ross, Jr., Secretary 


\begin{abstract}
The purpose of rigid-body registration is to find a rotation and translation which transforms one coordinate frame to another. The procedure requires measurement of common points (fiducials) in both frames. Noise and possible bias in the acquired locations of fiducials adversely affect the quality of registration. While the influence of noise can be mitigated by taking repeated measurements, bias remains in the mean locations of fiducials and distorts the rigid-body condition. The condition may be also violated when fiducials are attached to a non-rigid object. We present a numerical method which calculates corrections in the locations of measured fiducials and leads to the restoration of the rigid-body condition. Once the registration transformation is calculated using the corrected fiducials, it can be used to transform other points which are measured only in one coordinate frame (target points). We estimate the corrections in these points by linearly interpolating the corrections from neighboring fiducials. After interpolated corrections are added to the target locations, there was a decrease in the target registration error. The proposed method was evaluated using three different datasets acquired with three different instruments.
\end{abstract}

\title{
Keywords:
}

Bias, fiducials, noise, point-based rigid-body registration, target registration error. 


\section{Contents}

ABSTRACT ... i

1 INTRODUCTION........

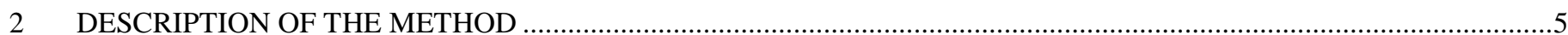

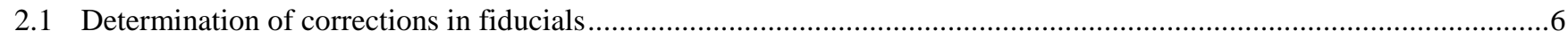

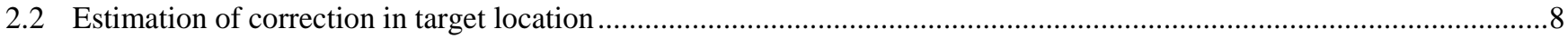

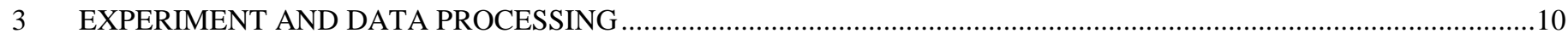

$4 \quad$ RESULTS

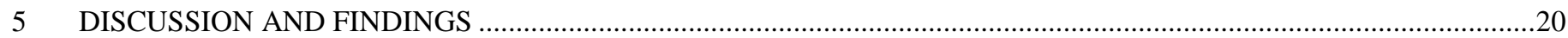

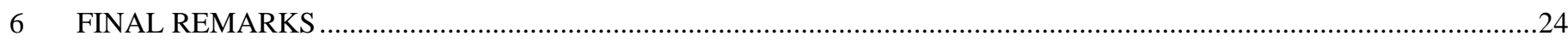

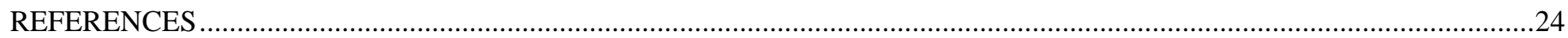




\section{INTRODUCTION}

Registration is needed when points measured in one coordinate frame are needed in another frame. In order to use the data, a transformation between both frames has to be determined. We call the first coordinate frame, from which points are transformed, the working frame, and the second frame, to which points are transformed, the destination frame. Rigid-body registration is the most frequently used method to determine the transformation, and it assumes that the distance between any two points $\left(\boldsymbol{X}_{i}, \boldsymbol{X}_{j}\right)$ in the working frame is equal to the distance between the same two points $\left(\boldsymbol{Y}_{i}, \boldsymbol{Y}_{j}\right)$ in the destination frame. Due to noise and possible bias in the acquired data, the rigid-body condition

$$
\left\|\boldsymbol{X}_{i, n}-\boldsymbol{X}_{j, n}\right\|=\left\|\boldsymbol{Y}_{i, n}-\boldsymbol{Y}_{j, n}\right\|
$$

is not satisfied in n-th noisy measurement. Even when the measurement is free of noise and bias, departure from the rigid-body condition may occur when fiducials used for registration are attached to an object which is not perfectly rigid. An example is when a pose of an object, such as an airplane wing, is measured from a set of markers attached to its surface. The wing may flex due to gravity, and the distances between markers will differ from their nominal values whenever the wing is moved. This will affect the pose of the object as the assumption of rigid-body is no longer valid. In this report, we will demonstrate experimental examples of restoring rigid-body condition only for the first scenario, where the inequality in Eq. (1) is caused by the measuring instrument (i.e., its noise and bias); however, the method introduced in this paper is also applicable to the second scenario with semi-rigid objects.

The distance preserving transformation between two frames consists of rotation $\boldsymbol{R}$ and translation $\boldsymbol{\tau}$ and can be obtained by minimizing the following error function

$$
E\left(\boldsymbol{R}_{n}, \boldsymbol{\tau}_{n}\right)=\frac{1}{J} \sum_{j=1}^{J}\left\|\boldsymbol{R}_{n} \boldsymbol{X}_{j, n}+\boldsymbol{\tau}_{n}-\boldsymbol{Y}_{j, n}\right\|^{2}
$$


Two sets of $J$ corresponding points used for registration, $\{\boldsymbol{X}\}_{J}$ in the working and $\{\boldsymbol{Y}\}_{J}$ in the destination frame, are called fiducials. A larger residual value of the error function $E$ is an indicator of more severe violation of the rigid-body condition caused by noise and possible bias in the measured locations of the fiducials. The influence of noise can be substantially reduced by taking many repeated measurements of $\{\boldsymbol{X}\}_{J, n},\{\boldsymbol{Y}\}_{J, n}(n=$ $1, \ldots, N)$ and using the mean values in Eq. (2)

$$
\overline{\boldsymbol{X}}_{j}=\frac{1}{N} \sum_{n=1}^{N} \boldsymbol{X}_{j, n}, \overline{\boldsymbol{Y}}_{j}=\frac{1}{N} \sum_{n=1}^{N} \boldsymbol{Y}_{j, n}
$$

For a measurement with no bias, such a noise filtering technique should bring the residual value of the error function $E$ close to zero and should yield a more accurate transformation $\{\boldsymbol{R}, \boldsymbol{\tau}\}$ (this statement also applies if there is a non-zero constant bias in either of the datasets). However, this technique does not remove position dependent bias from the measured locations of fiducials and cannot decrease $E$ below $E_{\min }$ defined as

$$
E_{\min }\left(\{\overline{\boldsymbol{X}}\}_{J},\{\overline{\boldsymbol{Y}}\}_{J}\right)=\frac{1}{J} \sum_{j=1}^{J} L_{\max , j}^{2}
$$

where

$$
L_{\max , j}=\frac{1}{2} \max \left\{\left|L_{i, j}\right| ; i=1, \ldots, J\right\}
$$

and

$$
L_{i, j}=\left\|\overline{\boldsymbol{X}}_{i}-\overline{\boldsymbol{X}}_{j}\right\|-\left\|\overline{\boldsymbol{Y}}_{i}-\overline{\boldsymbol{Y}}_{j}\right\|,
$$



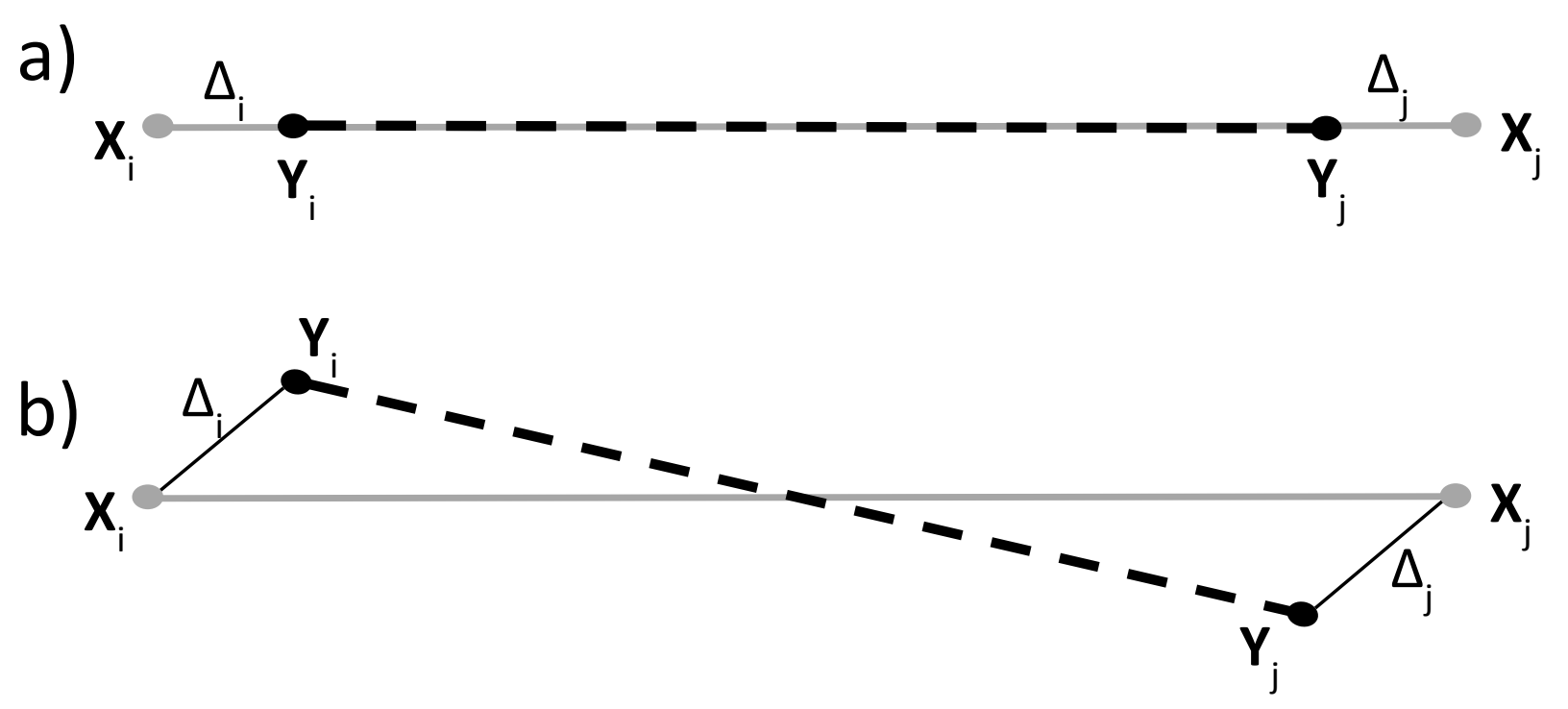

Figure 1. Schematic explanation of equations (4-6): a) the smallest possible value of the sum $\Delta_{i}^{2}+\Delta_{j}^{2}$ is when $\Delta_{i}=\Delta_{j}=L_{i j} / 2$ and the two pair of fiducials are collinear; b) for an arbitrary orientation of fiducials, the sum must be greater than $1 / 2 L_{i j}^{2}$ and it has to be true for any pair of fiducials $(i, j)$.

as explained schematically in Figure 1.

Once the registration is completed and the transformation $\{\boldsymbol{R}, \boldsymbol{\tau}\}$ determined, it can be used to transform other points $\left\{\boldsymbol{T}_{X}\right\}$ from the working to the destination frame. These points, called targets points, are not used for registration and, in principle, they are measured only in the working frame. Sometimes, in practical applications, only a subset of common points measured in both frames is used for registration and then, the remaining common points can be used to evaluate the quality of the registration. In research studies like this one, a separate set of corresponding targets $\left\{\boldsymbol{T}_{Y}\right\}$ is measured in the destination frame so that the quality of registration can be quantified.

In [1], a method was proposed to evaluate bias in the locations of fiducials. The method relies on evaluating the mean vector (averaged over many noisy measurements) of the Fiducial Registration Error at each fiducial, $\boldsymbol{F R E}\left(\boldsymbol{X}_{j}\right)$. The method enables one to draw some interesting general conclusions. For example, for the case of homogeneous bias $\boldsymbol{\varepsilon}$ (identical at each fiducial location), mean $\left\langle\boldsymbol{F} \boldsymbol{R E}\left(\boldsymbol{X}_{j}\right)\right\rangle=\mathbf{0}$ for all $j$ but the mean Target 
Registration Error $\left\langle\boldsymbol{T} \boldsymbol{R E}\left(\boldsymbol{T}_{X}\right)\right\rangle=-\boldsymbol{\varepsilon}$, see Eq. (27) and Eq. (31) in [1]. For the more general case of nonhomogeneous bias $\boldsymbol{\varepsilon}_{j}$ (different at each fiducial $\boldsymbol{X}_{j}$ ) the method yields a set of dependent linear equations which cannot be solved, see Eq. (23) in [1]. In order to overcome this problem, a set of $J$ targets without bias $\left\{\boldsymbol{T}_{X}\right\}_{J}$ is required, compare Eq. (12) and Eq. (28) in [1]. However, this requirement may not be realistic because if the measurements from an instrument contain a bias, the bias will exist in both the measurements of the fiducials and of the targets. Since the method in [1] assumes that the target measurements do not contain any bias, it does not provide an estimate of the bias of the target location. In addition, the uncertainty of the target measured in the working frame $\boldsymbol{T}_{X}$ is not considered and the method assumes that uncertainty in the transformed target $\boldsymbol{R} \boldsymbol{T}_{X}+\boldsymbol{\tau}$ comes only from the uncertainties of fiducials $\{\boldsymbol{X}\}_{J},\{\boldsymbol{Y}\}_{J}$ propagated to the transformation $\{\boldsymbol{R}, \boldsymbol{\tau}\}$ Similarly, the systematic error (defined as the mean difference between the transformed target and corresponding target in the destination frame $\boldsymbol{T}_{Y}$ ) is attributed only to the bias in the fiducials (i.e., not to the combined bias from the fiducials and target $\boldsymbol{T}_{X}$ ).

Even if noise is filtered out from the fiducials in the working and reference frames, the rigid-body condition can still be violated because of bias in either the working or destination frame. In this paper, we present a method which restores the rigid-body condition by applying corrections to the locations of fiducials in the working frame only. The aim of the proposed procedure is to restore the rigid-body condition and assure that fiducials in the working frame can be mapped exactly onto the corresponding fiducials in the destination frame. Although the bias may only be present in the destination frame, the method described in this paper works as if the bias was exclusively in the working frame and treats the mean locations of the fiducials in the destination frame as "truth". The method compensates for such understood "bias" in the fiducials $\{\boldsymbol{X}\}_{J}$. Then, the resulting residual value of the error function $E$ is reduced to zero (within numerical round-off error). Once correction $\boldsymbol{\varepsilon}_{j}$ at each fiducial $\boldsymbol{X}_{\boldsymbol{j}}$ is evaluated, the correction at any target location $\boldsymbol{T}_{X}$ is approximated by selecting four fiducials $\left\{\boldsymbol{X}_{j 1}, \boldsymbol{X}_{j 2}, \boldsymbol{X}_{j 3}, \boldsymbol{X}_{j 4}\right\}$, which form the smallest tetrahedron containing $\boldsymbol{T}_{X}$, and by linearly interpolating 
the four corrections $\left\{\varepsilon_{j 1}, \varepsilon_{j 2}, \varepsilon_{j 3}, \varepsilon_{j 4}\right\}$. This allows correction in the target location in the working frame and reduction of the target registration error TRE.

The method is demonstrated on data acquired by three different instruments: 1) motion tracking, System A; 2) large scale metrology, System B; and 3) laser tracker, LT. The data acquired by System A are characterized by relatively small noise but large bias. The data collected with System B have large noise but small bias. The data obtained with LT are considered as noise and bias free for the purpose of this study, see [2] for details. The obtained results show substantial improvement in the registration quality for System A registered to LT and modest improvement for System B registered to LT as quantified by TRE.

\section{DESCRIPTION OF THE METHOD}

This section describes a method to determine the correction in the fiducial location and a method used to estimate the correction in the target location. To calculate the correction in the fiducial location, both sets of fiducials are used to determine correction $\boldsymbol{\varepsilon}_{j}$ in the working frame for each $j=1, \ldots, J$. The correction of the fiducial locations is then used to estimate correction in the target location. The trilinear interpolation scheme applicable to an unorganized set of points is introduced and is used to estimate the correction in the target location as measured in the working frame.

The method assumes that a sufficiently large number $N$ of repeat measurements of noisy fiducials $\{\boldsymbol{X}\}_{J, n}$, $\{\boldsymbol{Y}\}_{J, n}$, and targets $\left\{\boldsymbol{T}_{X}\right\}_{n}$ can be acquired under fixed experimental conditions $(n \leq N)$ so that the resulting mean locations $\{\overline{\boldsymbol{X}}\}_{J},\{\overline{\boldsymbol{Y}}\}_{J}$, and $\left\{\overline{\boldsymbol{T}}_{X}\right\}$ are affected by bias only. $N$ may be deemed as sufficiently large if the difference $L_{i, j}$ in Eq. (6) does not change much with the increased number of repeats $N$, for example: $\left|L_{i, j}(N)-L_{i, j}(N / 2)\right| / L_{i, j}(N)<0.01$ where $L_{i, j}(N)$ is calculated from the mean locations obtained from $N$ repeats. 


\subsection{Determination of corrections in fiducials}

In order to restore the rigid-body condition, locations of fiducials $\{\overline{\boldsymbol{X}}\}_{J}$ are modified by unknown correction vectors $\{\boldsymbol{\varepsilon}\}_{J}$ such that

$$
\left\|\left(\overline{\boldsymbol{X}}_{i}-\boldsymbol{\varepsilon}_{i}\right)-\left(\overline{\boldsymbol{X}}_{j}-\boldsymbol{\varepsilon}_{j}\right)\right\|^{2}=\left\|\overline{\boldsymbol{Y}}_{i}-\overline{\boldsymbol{Y}}_{j}\right\|^{2},
$$

where $1 \leq i<j \leq J$. We assume that the rigid-body condition is only slightly violated in Eq. (1) and $\left\|\varepsilon_{i}\right\|,\left\|\varepsilon_{j}\right\| \ll\left\|\bar{X}_{i}-\bar{X}_{j}\right\|$ in Eq. (7). Therefore, we use linear approximation, where the squared terms $\left\|\varepsilon_{i}\right\|^{2}$ and $\left\|\varepsilon_{j}\right\|^{2}$ are ignored and Eq. (7) can be written as

$$
\left(\overline{\boldsymbol{X}}_{i}-\overline{\boldsymbol{X}}_{j}\right) \cdot\left(\boldsymbol{\varepsilon}_{i}^{\prime}-\boldsymbol{\varepsilon}_{j}^{\prime}\right)=\frac{1}{2}\left(\left\|\overline{\boldsymbol{Y}}_{i}-\overline{\boldsymbol{Y}}_{j}\right\|^{2}-\left\|\overline{\boldsymbol{X}}_{i}-\overline{\boldsymbol{X}}_{j}\right\|^{2}\right),
$$

where $\cdot$ is the dot product and $\left\{\boldsymbol{\varepsilon}^{\prime}\right\}_{J}$ are approximate corrections satisfying the set of linear equations in Eq. (8).

In matrix notation, equation (8) written as

$$
\boldsymbol{A}^{\prime} \boldsymbol{\varepsilon}^{\prime}=\boldsymbol{b}^{\prime}
$$

needs to be solved for the unknown column vector $\boldsymbol{\varepsilon}^{\prime}$ of size $[3 \mathrm{~J}, 1]$

$$
\boldsymbol{\varepsilon}^{\prime}=\left[\varepsilon_{x, 1}^{\prime}, \varepsilon_{y, 1}^{\prime}, \varepsilon_{z, 1}^{\prime}, \quad \ldots \quad \varepsilon_{x, J}^{\prime}, \varepsilon_{y, J}^{\prime}, \varepsilon_{z, J}^{\prime}\right]^{T}
$$

The known column vector $\boldsymbol{b}^{\prime}$ of size $\left[N_{p}, 1\right]$ is equal to

$$
\boldsymbol{b}^{\prime}=\left[b_{1,2}, \ldots, b_{1, J}, b_{2,3}, \ldots, b_{2, J}, \ldots, b_{J-1, J}\right]^{T}
$$

where $N_{p}=J(J-1) / 2$ is the number of unique pairs $(i, j)$ and $b_{i, j}$ represents the right hand side of Eq. (8)

$$
b_{i, j}=\frac{1}{2}\left(\left\|\overline{\boldsymbol{Y}}_{i}-\overline{\boldsymbol{Y}}_{j}\right\|^{2}-\left\|\overline{\boldsymbol{X}}_{i}-\overline{\boldsymbol{X}}_{j}\right\|^{2}\right)
$$

Finally, matrix $\boldsymbol{A}^{\prime}$ in Eq. (9) has size of $\left[N_{p}, 3 \mathrm{~J}\right]$ and consists mostly of zero elements except for six non-zero entries in each row as follows from Eq. (8). Linear equation Eq. (9) can be solved for $\boldsymbol{\varepsilon}^{\prime}$ using Singular Value Decomposition (SVD), but the solution is not unique: if $\boldsymbol{\varepsilon}_{j}^{\prime}$ is a solution then $\boldsymbol{\varepsilon}_{j}^{\prime}+\boldsymbol{v}$ is also a solution where $\boldsymbol{v}$ 
is an arbitrary $3 \mathrm{D}$ vector, the same for all $j \leq J$. This step may be iterated once more to get a better estimate of the correction by substituting $\overline{\boldsymbol{X}}_{i}-\boldsymbol{\varepsilon}_{i}^{\prime}$ for $\overline{\boldsymbol{X}}_{i}$ in Eq. (8) and solving the equation for the unknown $\boldsymbol{\varepsilon}_{i}^{\prime \prime}$

$$
\left[\left(\overline{\boldsymbol{X}}_{i}-\boldsymbol{\varepsilon}_{i}^{\prime}\right)-\left(\overline{\boldsymbol{X}}_{j}-\boldsymbol{\varepsilon}_{j}^{\prime}\right)\right] \cdot\left(\boldsymbol{\varepsilon}_{i}^{\prime \prime}-\boldsymbol{\varepsilon}_{j}^{\prime \prime}\right)=\frac{1}{2}\left(\left\|\overline{\boldsymbol{Y}}_{i}-\overline{\boldsymbol{Y}}_{j}\right\|^{2}-\left\|\left(\overline{\boldsymbol{X}}_{i}-\boldsymbol{\varepsilon}_{i}^{\prime}\right)-\left(\overline{\boldsymbol{X}}_{j}-\boldsymbol{\varepsilon}_{j}^{\prime}\right)\right\|^{2}\right)
$$

or, in matrix form

$$
\boldsymbol{A}^{\prime \prime} \varepsilon^{\prime \prime}=\boldsymbol{b}^{\prime \prime}
$$

analogous to Eqs.(8-11). After solving Eq. (14) (again, the solution $\boldsymbol{\varepsilon}^{\prime \prime}$ is not unique), the resulting estimate of the correction $\boldsymbol{\varepsilon}_{j}$ and the corrected location of fiducial $\widetilde{\boldsymbol{X}}_{j}$ in the working frame are equal

$$
\varepsilon_{j}=\varepsilon_{j}^{\prime}+\varepsilon_{j}^{\prime \prime}, \quad \widetilde{X}_{j}=\bar{X}_{j}-\varepsilon_{j}
$$

No further iterations are needed as the convergence is achieved in two steps except for the situation when all the points $\{\overline{\boldsymbol{X}}\}_{J}$ in the working frame are located on a plane perpendicular to any one of the three axes of the Cartesian coordinate frame. For example, if the $z$ component of each vector $\overline{\boldsymbol{X}}_{j}$ is the same, then the $z$ component of each correction vector $\boldsymbol{\varepsilon}_{j}^{\prime}$ is zero and the convergence is not achieved.

Next, registration between the corrected fiducials $\{\widetilde{\boldsymbol{X}}\}_{J}$ in the working frame and fiducials $\{\overline{\boldsymbol{Y}}\}_{J}$ in the destination frame can be performed. Then, the resulting residual value of the error function $E$ should be practically reduced to zero and the corresponding transformation $\{\boldsymbol{R}, \boldsymbol{\tau}\}$ should map $\{\widetilde{\boldsymbol{X}}\}_{J}$ exactly onto $\{\overline{\boldsymbol{Y}}\}_{J}$ because the rigid-body condition

$$
\left\|\widetilde{\boldsymbol{X}}_{i}-\widetilde{\boldsymbol{X}}_{j}\right\|=\left\|\overline{\boldsymbol{Y}}_{i}-\overline{\boldsymbol{Y}}_{j}\right\|
$$

is now restored for all pairs of processed fiducials $(i, j)$.

As stated earlier, but which bears repetition, the restoration of rigid-body condition obtained by applying corrections $\{\boldsymbol{\varepsilon}\}_{J}$ is performed in the working frame. This, however, does not imply that $\{\overline{\boldsymbol{Y}}\}_{J}$ measured in the destination frame constitute ground truth. 


\subsection{Estimation of correction in target location}

Correction in the target location $\boldsymbol{\varepsilon}\left(\overline{\boldsymbol{T}}_{X}\right)$ cannot be achieved in the same way as corrections in fiducials because in most practical applications, the target $\left\{\overline{\boldsymbol{T}}_{Y}\right\}$ is not measured in the destination frame. Therefore, correction $\boldsymbol{\varepsilon}\left(\overline{\boldsymbol{T}}_{X}\right)$ is estimated by linear interpolation of previously calculated corrections in four nearby fiducials. In general, the set of $J$ fiducials $\{\overline{\boldsymbol{X}}\}_{J}$ is not acquired in a regular grid. Therefore, popular trilinear interpolation schemes requiring measured points located on a strictly regular 3D grid are not applicable [3]. We use tetrahedral linear interpolation illustrated schematically in Figure 2 and described in [4]. This kind of interpolation method was used to interpolate between discrete points in colorspace [5].

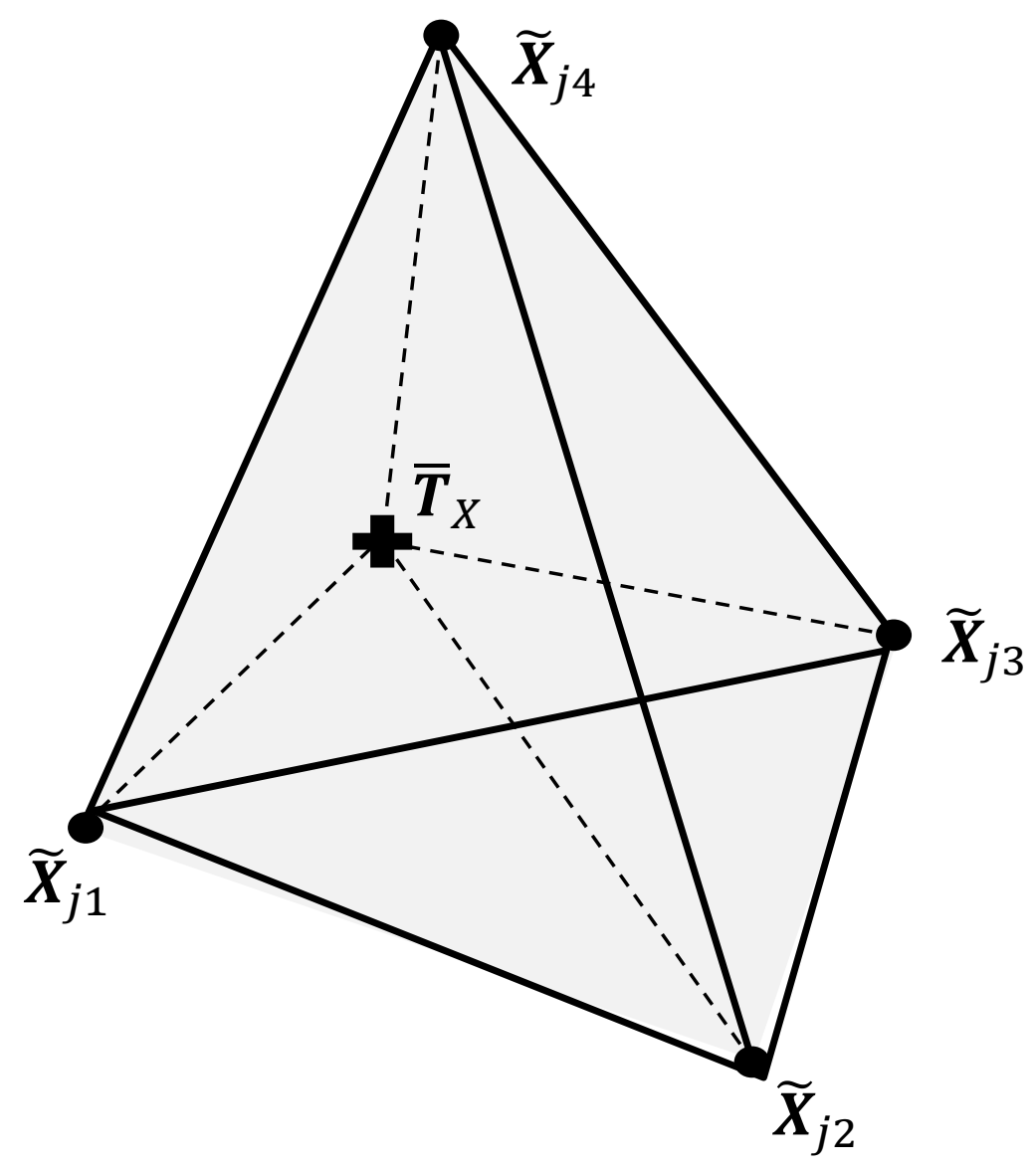

Figure 2. Tetrahedral linear interpolation. Unknown bias at target location $\boldsymbol{\varepsilon}\left(\overline{\boldsymbol{T}}_{X}\right)$ is linearly interpolated from the four biases calculated at four nearest fiducials $\boldsymbol{\varepsilon}\left(\widetilde{\boldsymbol{X}}_{k}\right)$, where $k \in\left\{j_{1}, j_{2}, j_{3}, j_{4}\right\}$. 
For a given target location $\overline{\boldsymbol{T}}_{X}$ in the working space, the four vertices of the tetrahedron $\left\{\widetilde{\boldsymbol{X}}_{j 1}, \widetilde{\boldsymbol{X}}_{j 2}, \widetilde{\boldsymbol{X}}_{j 3}, \widetilde{\boldsymbol{X}}_{j 4}\right\}$ containing $\overline{\boldsymbol{T}}_{X}$ are selected in the following way. For each $j \leq J$, distances $d_{j}=\left\|\overline{\boldsymbol{T}}_{X}-\widetilde{\boldsymbol{X}}_{j}\right\|$ are calculated and sorted in ascending order where $\widetilde{\boldsymbol{X}}_{j}$ is the corrected fiducial location compensated by the correction calculated using Eq. (15). The first three smallest distances $\left\{d_{j 1}, d_{j 2}, d_{j 3}\right\}$ define three vertices $\left\{\widetilde{\boldsymbol{X}}_{j 1}, \widetilde{\boldsymbol{X}}_{j 2}, \widetilde{\boldsymbol{X}}_{j 3}\right\}$ of the sought tetrahedron. Then, the fiducial $\widetilde{\boldsymbol{X}}_{i 4}$ corresponding to the fourth smallest distance $d_{i 4}$ is checked as a possible candidate for the remaining vertex. The candidate is accepted if the resulting tetrahedron passes the test for containing target point $\overline{\boldsymbol{T}}_{X}$. The test is based on the observation that the sum of volumes of four tetrahedrons (where one of the vertices is the target point) must be equal to the volume of the tetrahedron formed by the four fiducials. Thus, the following condition has to be satisfied

$$
\sum_{k=1}^{4} w_{k}=1
$$

where $w_{k}$ is the ratio of two tetrahedron volumes

$$
w_{k}=\operatorname{vol}\left(\left\{\widetilde{\boldsymbol{X}}, \overline{\boldsymbol{T}}_{\boldsymbol{X}}\right\}_{k}\right) / \operatorname{vol}\left(\left\{\widetilde{\boldsymbol{X}}_{j 1}, \widetilde{\boldsymbol{X}}_{j 2}, \widetilde{\boldsymbol{X}}_{j 3}, \widetilde{\boldsymbol{X}}_{i 4}\right\}\right)
$$

and $\left\{\widetilde{\boldsymbol{X}}, \boldsymbol{T}_{X}\right\}_{k}$ denotes a set of four points $\left\{\widetilde{\boldsymbol{X}}_{j 1}, \widetilde{\boldsymbol{X}}_{j 2}, \widetilde{\boldsymbol{X}}_{j 3}, \widetilde{\boldsymbol{X}}_{i 4}\right\}$ with $k$-th point substituted by $\overline{\boldsymbol{T}}_{X}, k \leq 4$. For example, $\left\{\widetilde{\boldsymbol{X}}, \overline{\boldsymbol{T}}_{X}\right\}_{1}=\left\{\overline{\boldsymbol{T}}_{X}, \widetilde{\boldsymbol{X}}_{j 2}, \widetilde{\boldsymbol{X}}_{j 3}, \widetilde{\boldsymbol{X}}_{i 4}\right\}$ and $\left\{\widetilde{\boldsymbol{X}}, \overline{\boldsymbol{T}}_{X}\right\}_{4}=\left\{\widetilde{\boldsymbol{X}}_{j 1}, \widetilde{\boldsymbol{X}}_{j 2}, \widetilde{\boldsymbol{X}}_{j 3}, \overline{\boldsymbol{T}}_{X}\right\}$. The volume of the tetrahedron formed by four points $\left\{\boldsymbol{P}_{1}, \boldsymbol{P}_{2}, \boldsymbol{P}_{3}, \boldsymbol{P}_{4}\right\}$ is the absolute value of the scalar triple product

$$
\operatorname{vol}\left(\left\{\boldsymbol{P}_{1}, \boldsymbol{P}_{2}, \boldsymbol{P}_{3}, \boldsymbol{P}_{4}\right\}\right)=\frac{1}{6}\left|\left(\boldsymbol{P}_{2}-\boldsymbol{P}_{1}\right) \cdot\left[\left(\boldsymbol{P}_{3}-\boldsymbol{P}_{1}\right) \times\left(\boldsymbol{P}_{4}-\boldsymbol{P}_{1}\right)\right]\right|
$$

If the fiducial $\widetilde{\boldsymbol{X}}_{i 4}$ does not satisfy Eq. (17), i.e., $\sum_{k=1}^{4} w_{k}>1$, then the target point $\overline{\boldsymbol{T}}_{X}$ is not inside the tetrahedron $\left\{\widetilde{\boldsymbol{X}}_{j 1}, \widetilde{\boldsymbol{X}}_{j 2}, \widetilde{\boldsymbol{X}}_{j 3}, \widetilde{\boldsymbol{X}}_{i 4}\right\}$ and the next candidate point $\widetilde{\boldsymbol{X}}_{i 5}$ is checked and the process continues until the first fiducial $\widetilde{\boldsymbol{X}}_{i m}$ satisfies Eq. (17) where $\widetilde{\boldsymbol{X}}_{i m}$ corresponds to the distance $d_{i m}$ on a sorted list of distances $\left\{d_{j 1}, d_{j 2}, \ldots, d_{i m}, \ldots\right\}$. Then, $\widetilde{\boldsymbol{X}}_{j 4}=\widetilde{\boldsymbol{X}}_{i m}$ and the resulting tetrahedron $\left\{\widetilde{\boldsymbol{X}}_{j 1}, \widetilde{\boldsymbol{X}}_{j 2}, \widetilde{\boldsymbol{X}}_{j 3}, \widetilde{\boldsymbol{X}}_{j 4}\right\}$ contains $\overline{\boldsymbol{T}}_{X}$ and has the property that the following sum

$$
\sum_{k=1}^{4}\left\|\overline{\boldsymbol{T}}_{X}-\widetilde{\boldsymbol{X}}_{k}\right\|, k \in\left\{j_{1}, j_{2}, j_{3}, j_{4}\right\}
$$


is the smallest from all possible corrected fiducials $\{\widetilde{\boldsymbol{X}}\}_{J}$. Once the tetrahedron with this property is identified for a given target point $\overline{\boldsymbol{T}}_{X}$, the correction $\boldsymbol{\varepsilon}\left(\overline{\boldsymbol{T}}_{X}\right)$ can be estimated as the weighted mean of corrections $\boldsymbol{\varepsilon}_{k}$ calculated using Eq. (15) for the $k$-th fiducial

$$
\boldsymbol{\varepsilon}\left(\overline{\boldsymbol{T}}_{X}\right)=\sum_{k=1}^{4} w_{k} \boldsymbol{\varepsilon}_{k}, k \in\left\{j_{1}, j_{2}, j_{3}, j_{4}\right\}
$$

with weights $w_{k}$ calculated using Eq. (18). Finally, the corrected target location $\widetilde{\boldsymbol{T}}_{X}$ in the working frame can be determined as

$$
\widetilde{\boldsymbol{T}}_{X}=\overline{\boldsymbol{T}}_{X}-\varepsilon\left(\overline{\boldsymbol{T}}_{X}\right)
$$

It is easy to show that tetrahedral linear interpolation reduces to a simple 1D linear interpolation when the target $\overline{\boldsymbol{T}}_{X}$ is located on one of the tetrahedron edges and to a $2 \mathrm{D}$-simplex (triangular) interpolation when $\overline{\boldsymbol{T}}_{X}$ is on one of the tetrahedron faces. Correction $\boldsymbol{\varepsilon}\left(\overline{\boldsymbol{T}}_{X}\right)$ in Eq. (21) is a continuous function $\left(\mathrm{C}^{0}\right.$ class) of target location; it is differentiable but has discontinuous partial derivatives (not $\mathrm{C}^{1}$ class).

\section{EXPERIMENT AND DATA PROCESSING}

Locations of $3 \mathrm{D}$ points in a work volume (approximately $3 \mathrm{~m} \times 3 \mathrm{~m} \times 1.8 \mathrm{~m}$ ) were acquired by three instruments: 1) motion tracking, System A; 2) large scale metrology, System B; and 3) laser tracker, LT. Fiducials were measured on a semi-regular grid of $5 \times 5 \times 5$ points; in addition, $K=16$ target points scattered randomly in the work volume were also acquired. Measurement of each point (fiducial and target) were repeated $N=200$ times for Systems A and B; no repeats were obtained with LT as the noise level for laser tracker was negligible compared to the noise of Systems A and B. Care was taken to ensure that all three instruments measured the same physical point in the work volume, see [2] for details.

Repeated measurements taken with Systems A or B were used to calculate the corresponding mean locations of fiducials $\{\overline{\boldsymbol{X}}\}_{J}$ and targets $\left\{\overline{\boldsymbol{T}}_{X}\right\}_{K}$ in the working frame. Two registrations were investigated: coordinate frame A to LT and B to LT. Accordingly, the need for corrections in A or B was revealed by building histograms 
of differences $L_{i, j}$ calculated using Eq. (6) for all possible pairs $1 \leq i<j \leq J$. In addition, for each mean $k$-th target location $\overline{\boldsymbol{T}}_{X, k}$ the degree of departure from the rigid-body condition was quantified by calculating the distance $g(k, j)$

$$
g(k, j)=\left\|\overline{\boldsymbol{T}}_{X, k}-\overline{\boldsymbol{X}}_{j}\right\|-\left\|\overline{\boldsymbol{T}}_{Y, k}-\overline{\boldsymbol{Y}}_{j}\right\|
$$

for all $J$ fiducials $\{\overline{\boldsymbol{X}}\}_{J},\{\overline{\boldsymbol{Y}}\}_{J}\left(\overline{\boldsymbol{T}}_{Y, k}\right.$ is $k$-th target measured in the destination frame).

For each $j$-th fiducial $\bar{X}_{j}$, corresponding corrections $\varepsilon_{j}=\varepsilon_{j}^{\prime}+\varepsilon_{j}^{\prime \prime}$ were calculated by solving Eq. (9) and Eq. (14). To track the progress of the two iterations of the procedure to calculate the correction, histograms of differences $L_{i, j}$ were calculated using Eq. (6) after each iteration, i.e., $\overline{\boldsymbol{X}}_{i}, \overline{\boldsymbol{X}}_{j}$ were replaced with $\overline{\boldsymbol{X}}_{i}-\boldsymbol{\varepsilon}_{i}^{\prime}, \overline{\boldsymbol{X}}_{j}-$ $\boldsymbol{\varepsilon}_{j}^{\prime}$ after the first iteration and with $\overline{\boldsymbol{X}}_{i}-\boldsymbol{\varepsilon}_{i}^{\prime}-\boldsymbol{\varepsilon}_{i}^{\prime \prime}, \overline{\boldsymbol{X}}_{j}-\boldsymbol{\varepsilon}_{j}^{\prime}-\boldsymbol{\varepsilon}_{j}^{\prime \prime}$ after the second iteration. Once all $J$ corrections $\{\boldsymbol{\varepsilon}\}_{J}$ at the fiducial locations were calculated, registration was performed based on $\{\overline{\boldsymbol{Y}}\}_{J}$ in the destination frame and the corrected locations of fiducials $\{\widetilde{\boldsymbol{X}}\}_{J}$ in the working frame. Since the rigid-body condition has been restored, the actual number $J^{*}$ of corrected fiducials used for registration $\left(3 \leq J^{*} \leq J\right)$ and their placement in the work volume is irrelevant as all subsets of the corrected fiducials are supposed to yield the same registration transformation $\{\boldsymbol{R}, \boldsymbol{\tau}\}$ (with the obvious restriction that not all selected registration points are collinear). Similar to $L_{i, j}$, the distances $g(k, j)$ are calculated using Eq. (23) for the original, uncorrected locations of the fiducials $\overline{\boldsymbol{X}}_{j}$ and $\overline{\boldsymbol{T}}_{X, k}$ as well for the corrected locations $\widetilde{\boldsymbol{X}}_{j}$ and $\widetilde{\boldsymbol{T}}_{X, k}$, as given by Eq. (15) and Eq. (22). Once the registration transformation $\{\boldsymbol{R}, \boldsymbol{\tau}\}$ is found, it can be applied to calculate the $k$-th target registration error $\operatorname{TRE}(k)$

$$
\operatorname{TRE}(k)=\left\|\boldsymbol{R} \overline{\boldsymbol{T}}_{X, k}+\boldsymbol{\tau}-\boldsymbol{T}_{Y, k}\right\|
$$

as well as the root mean square $R M S_{T}$ of $T R E(k)$

$$
R M S_{T}=\sqrt{\frac{1}{K} \sum_{k=1}^{K} T R E^{2}(k)} .
$$

The $T R E(k)$ and resulting $R M S_{T}$ were calculated using three different post-processing scenarios. In scenario 1, the original mean locations of fiducials $\{\overline{\boldsymbol{X}}\}_{J}$ and targets $\left\{\overline{\boldsymbol{T}}_{X}\right\}_{K}$ in the working frame were used. A subset of 
four fiducials was selected from all possible combinations of $J^{*}=4$ out of $J=125$ fiducials which yielded the smallest $R M S_{T}$, as described in [2]. Once the registration to the destination frame was performed, the resulting transformation was used to transform the uncorrected mean locations of targets $\left\{\overline{\boldsymbol{T}}_{X}\right\}_{K}$ from the working to the destination frame and $\operatorname{TRE}(k)$ and $R M S_{T}$ were calculated. In scenario 2, the same four fiducials as in scenario 1 were chosen from the fiducials whose locations were corrected $\{\widetilde{\boldsymbol{X}}\}_{J}$. Thus, the resulting transformation $\{\boldsymbol{R}, \boldsymbol{\tau}\}$ was slightly different than in 1) and again, it was applied to the uncorrected targets $\left\{\overline{\boldsymbol{T}}_{X}\right\}_{K}$ and $\operatorname{TRE}(k)$ and $R M S_{T}$ were calculated. Finally, in scenario 3, the same transformation $\{\boldsymbol{R}, \boldsymbol{\tau}\}$ as in scenario 2 was applied to the targets $\left\{\widetilde{\boldsymbol{T}}_{X}\right\}_{K}$ (modified by the tetragonal interpolated corrections) and $T R E(k)$ and $R M S_{T}$ were calculated.

\section{RESULTS}

Figure 3 shows histograms of $L_{i, j}$ calculated using Eq. (6) for the mean, uncorrected locations of fiducials $\overline{\boldsymbol{X}}_{j}$ acquired with System A (Figure 3a), corrected locations after the first iteration of the procedure by $\boldsymbol{\varepsilon}_{j}^{\prime}$ (Figure 3b), and after the second iteration corrected by the total bias $\varepsilon_{j}^{\prime}+\varepsilon_{j}^{\prime \prime}$ (Figure 3c), as given by Eq. (15). For $J=$ 125 fiducials, there are $J(J-1) / 2=7,750$ unique pairs of fiducials. Figure 4 contains similar histograms calculated for data acquired with System B. 

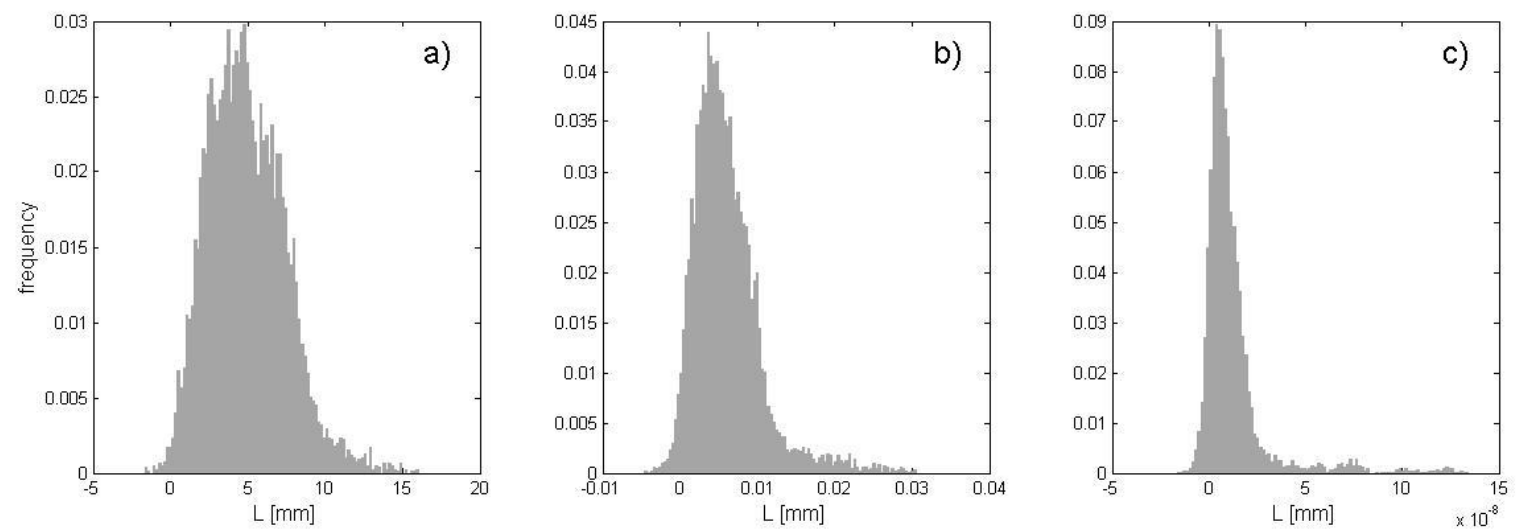

Figure 3. Histograms of $L_{i, j}$ calculated from data acquired by System A and LT: a) original, uncorrected locations of fiducials $\overline{\boldsymbol{X}}_{j}$; b) corrected location of fiducials $\overline{\boldsymbol{X}}_{j}-\boldsymbol{\varepsilon}_{j}^{\prime}$ after the first iteration of the procedure; c) final, corrected locations of fiducials $\widetilde{\boldsymbol{X}}_{j}$ given by Eq. (15) after the second iteration of the procedure.
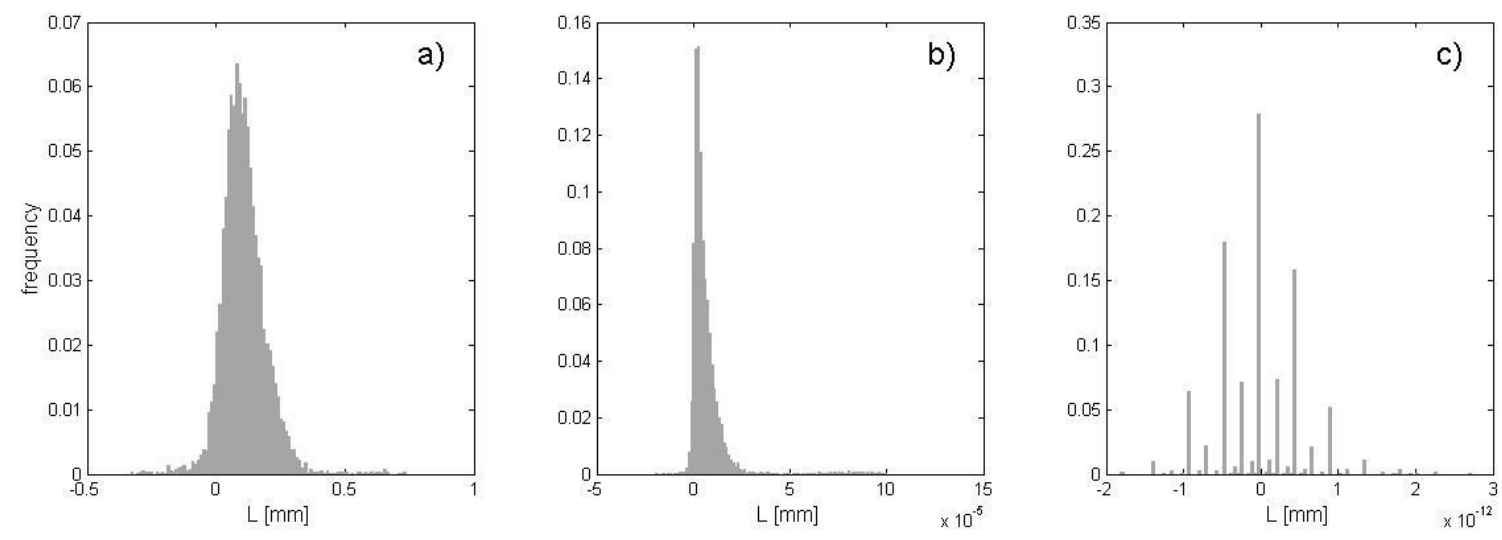

Figure 4. Histograms of $L_{i, j}$ calculated from data acquired by System B and LT: a) original, uncorrected locations of fiducials $\overline{\boldsymbol{X}}_{j}$; b) corrected location of fiducials $\overline{\boldsymbol{X}}_{j}-\boldsymbol{\varepsilon}_{j}^{\prime}$ after the first iteration of the procedure; c) final, corrected locations of fiducials $\widetilde{\boldsymbol{X}}_{j}$ given by Eq. (15) after the second iteration of the procedure.

Figure 5 shows the three Cartesian components of the correction vector $\boldsymbol{\varepsilon}_{j}$ calculated at mean fiducials $\overline{\boldsymbol{X}}_{j}$ for data acquired with System A and LT. Figure 6 shows vector $\boldsymbol{\varepsilon}_{j}$ calculated at mean fiducials $\overline{\boldsymbol{X}}_{j}$ for data acquired with System B and LT. 

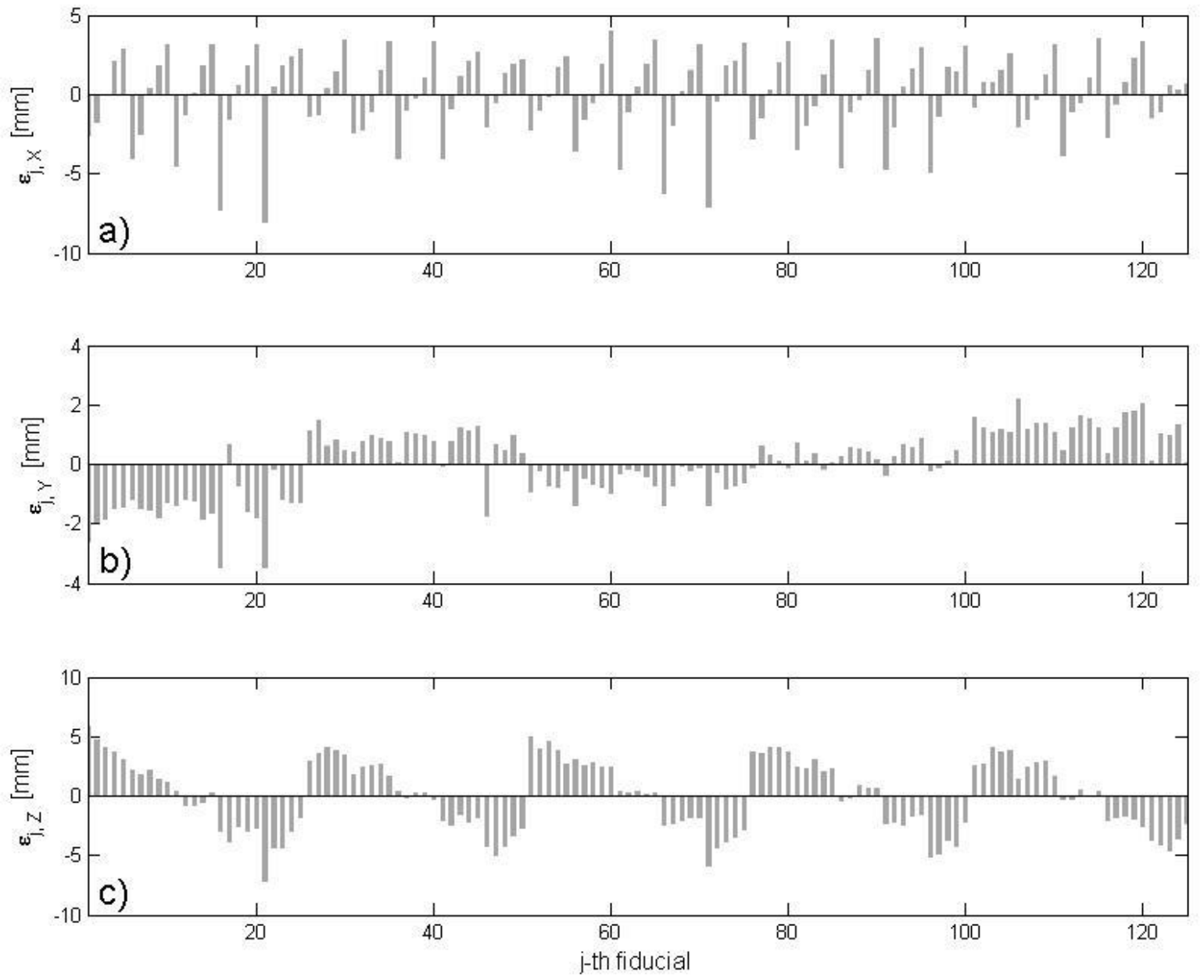

Figure 5. Components of the correction vectors $\boldsymbol{\varepsilon}_{j}$ calculated at mean locations of fiducials $\overline{\boldsymbol{X}}_{j}$ for data acquired with System A: a) $x$ component of correction $\boldsymbol{\varepsilon}_{j}$; b) $y$ component; c) $z$ component. 

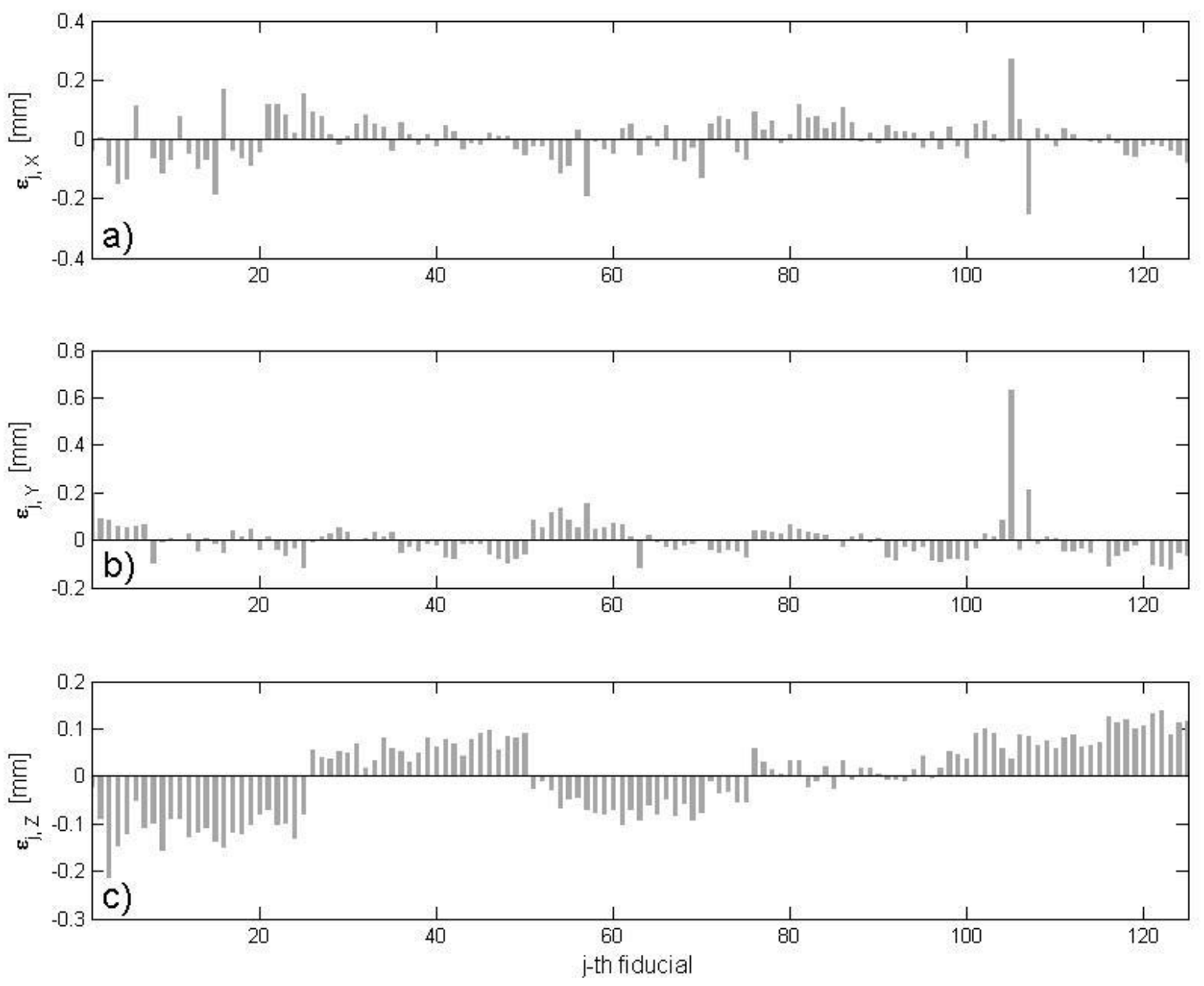

Figure 6. Components of the correction vectors $\boldsymbol{\varepsilon}_{j}$ calculated at mean locations of fiducials $\overline{\boldsymbol{X}}_{j}$ for data acquired with System B: a) $x$ component of correction $\boldsymbol{\varepsilon}_{j}$; b) $y$ component; c) $z$ component.

Figure 7 shows the magnitudes of the correction vector $\left\|\varepsilon_{j}\right\|$ calculated for data acquired with System A and displayed on a 3D graph. Figure 8 shows similar results for data acquired with System B. For easier comparison, both 3D graphs in Figure 7 and Figure 8 are displayed in the LT coordinate system (destination frame). 


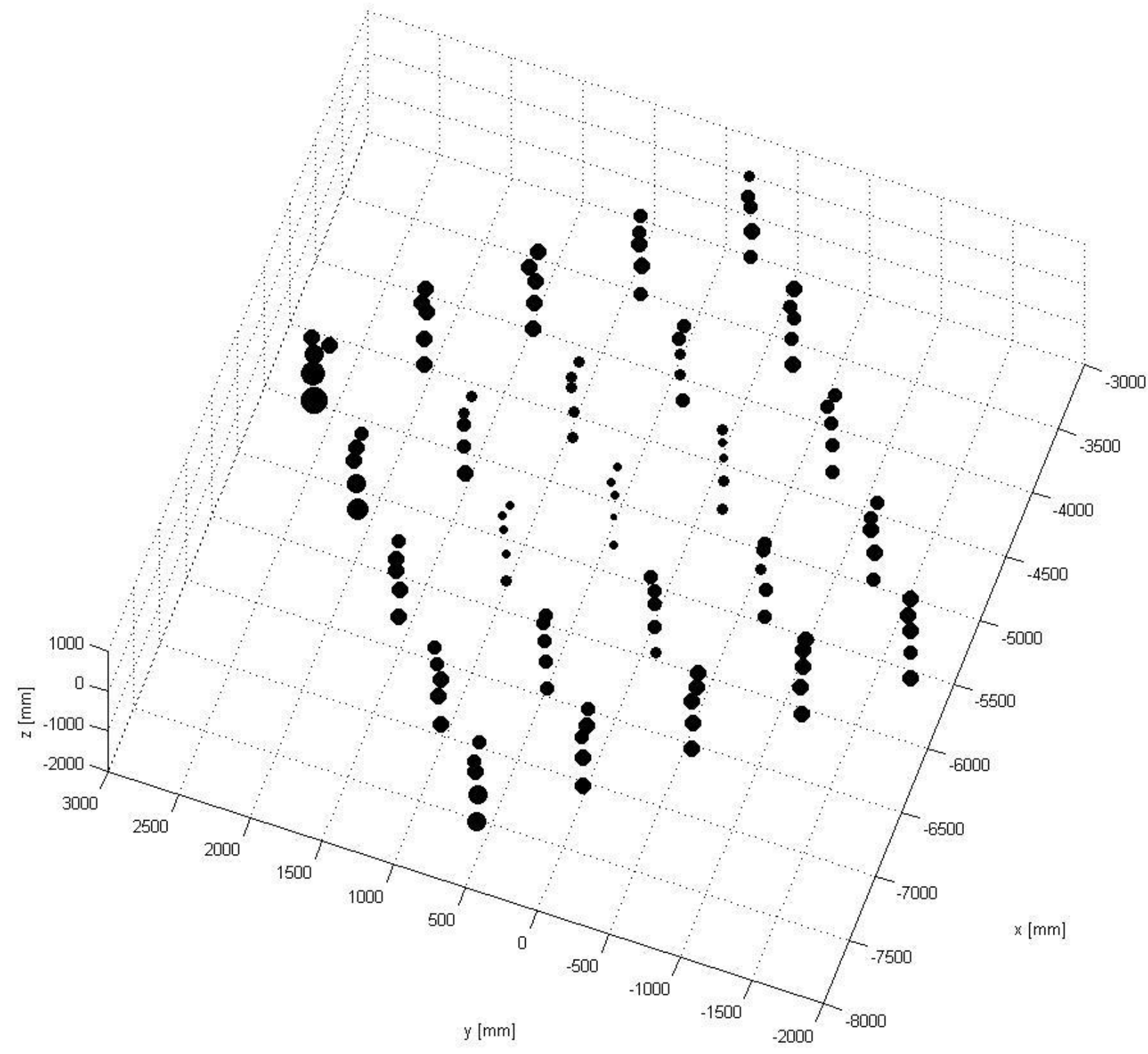

Figure 7. Magnitude of the correction vectors $\left\|\varepsilon_{j}\right\|$ calculated for data acquired with System A. The radius of the $j$-th sphere is proportional to $\left\|\varepsilon_{j}\right\|$, and the center of the sphere is located at $\overline{\boldsymbol{X}}_{j}$ transformed to the destination frame LT. 


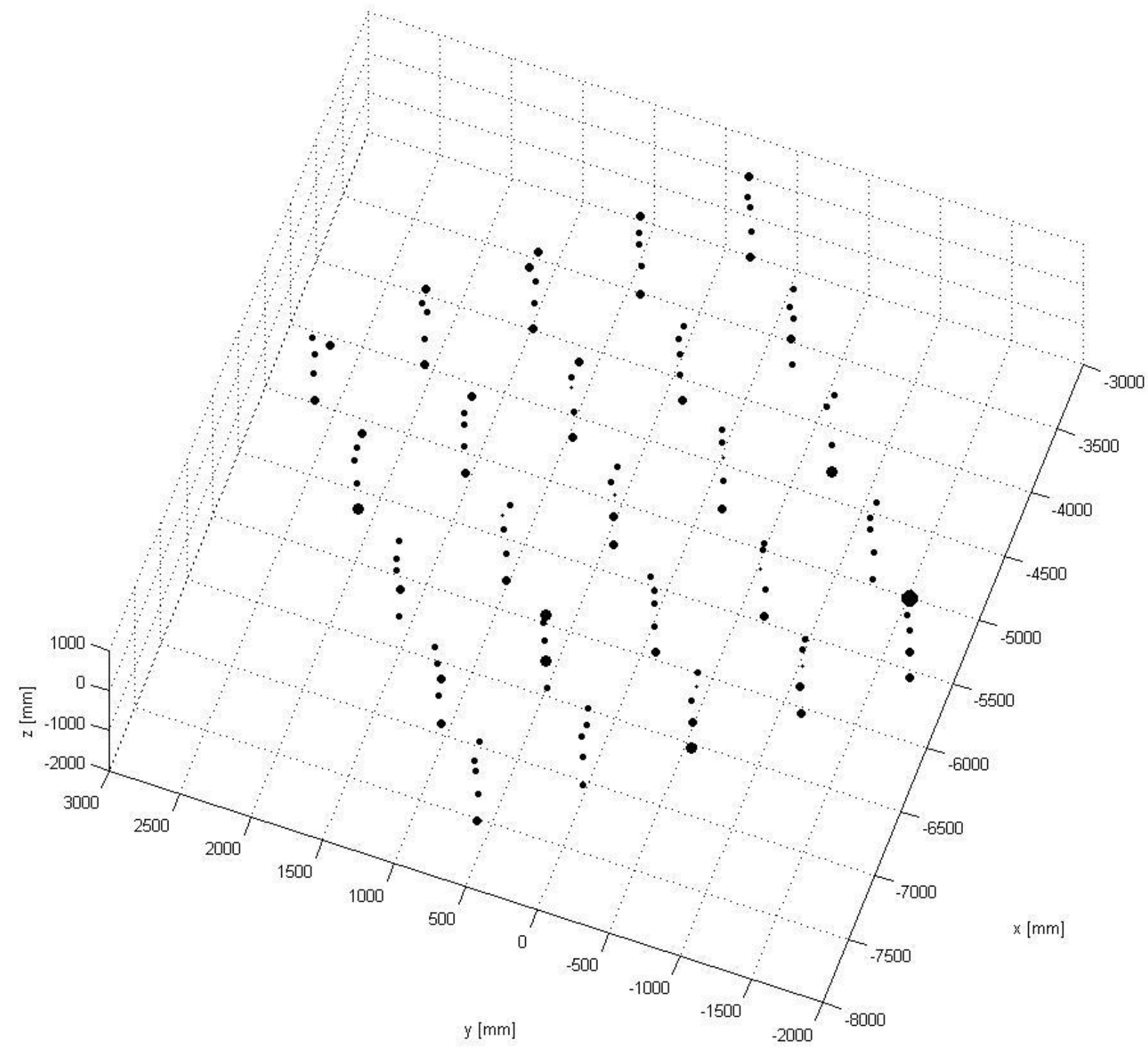

Figure 8. Magnitude of the correction vectors $\left\|\varepsilon_{j}\right\|$ calculated for data acquired with System B. The radius of the $j$-th sphere is proportional to $\left\|\boldsymbol{\varepsilon}_{j}\right\|$, and the center of the sphere is located at $\overline{\boldsymbol{X}}_{j}$ transformed to the destination frame LT.

Figure 9 shows the difference between the distances of the $k$-th target and the $j$-th fiducial in the working and the destination frame, as calculated using Eq. (23) for data acquired with System A and LT. Plotted are the 
mean difference $\langle g(k)\rangle$ averaged over all $J$ fiducials as well as the minimum and maximum difference. The plot in Figure 9a is based on the original, uncorrected locations of fiducials and target and Figure 9b is based on the corrected locations of fiducials and target. Figure 10 shows the similar results for data acquired with System B and LT.
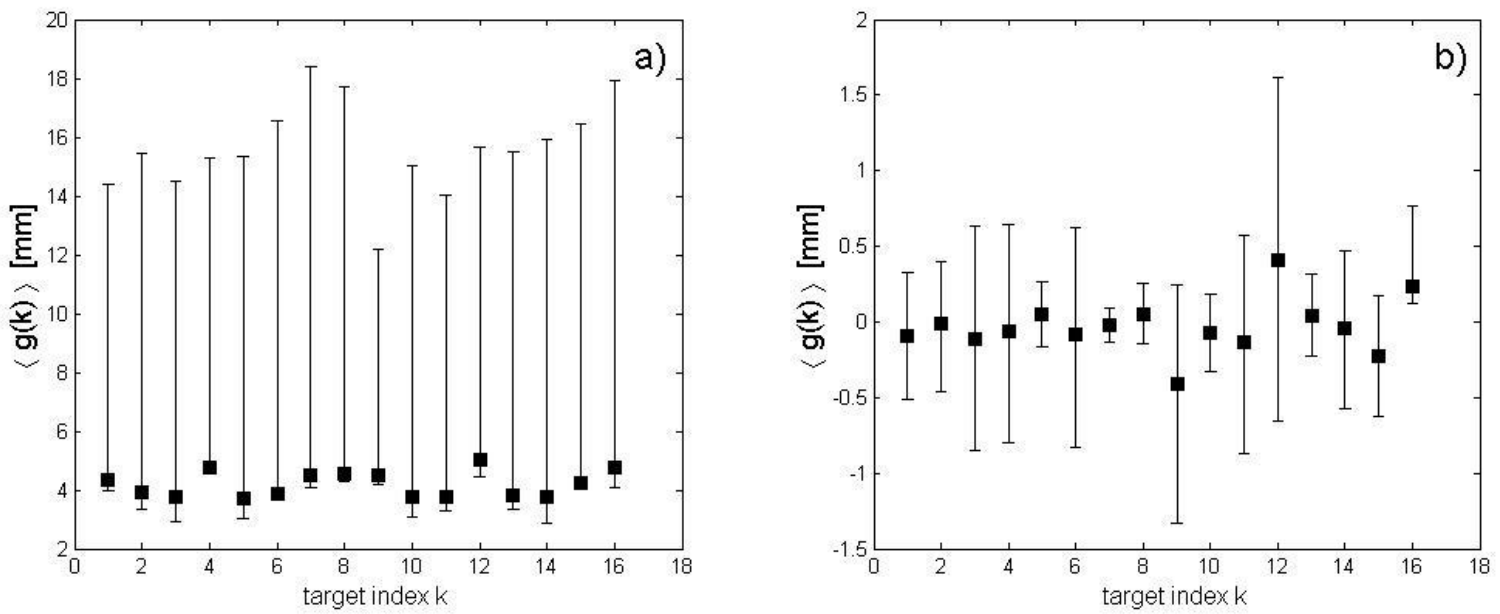

Figure 9. The difference $g$ for the $k$-th target for System A. Square markers correspond to the mean value calculated over all $J$ fiducials and error bars indicate the minimum and the maximum value of difference $g$. a) original, uncorrected locations of fiducials and target; b) corrected locations of fiducials and target.
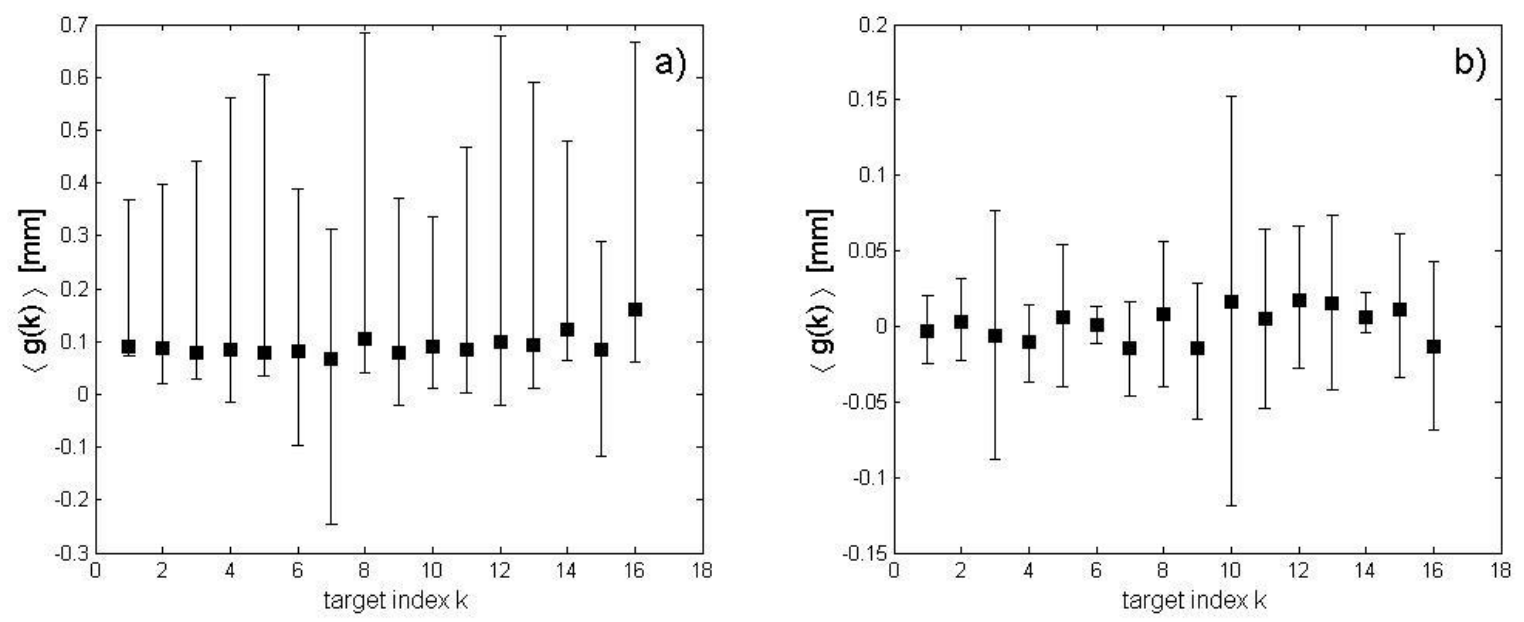

Figure 10. The difference $g$ for the $k$-th target for System B. Square markers correspond to the mean value calculated over all $J$ fiducials and error bars indicate the minimum and the maximum value of difference $g$. a) original, uncorrected locations of fiducials and target; b) corrected locations of fiducials and target. 
Target registration errors $\operatorname{TRE}(k)$ for the three post-processing scenarios 1,2, and 3 are plotted in Figure 11 and Figure 12 for data acquired with Systems A and B, respectively. Finally, Figure 13 shows the corresponding $R M S_{T}$ for the three scenarios for Systems A and B.

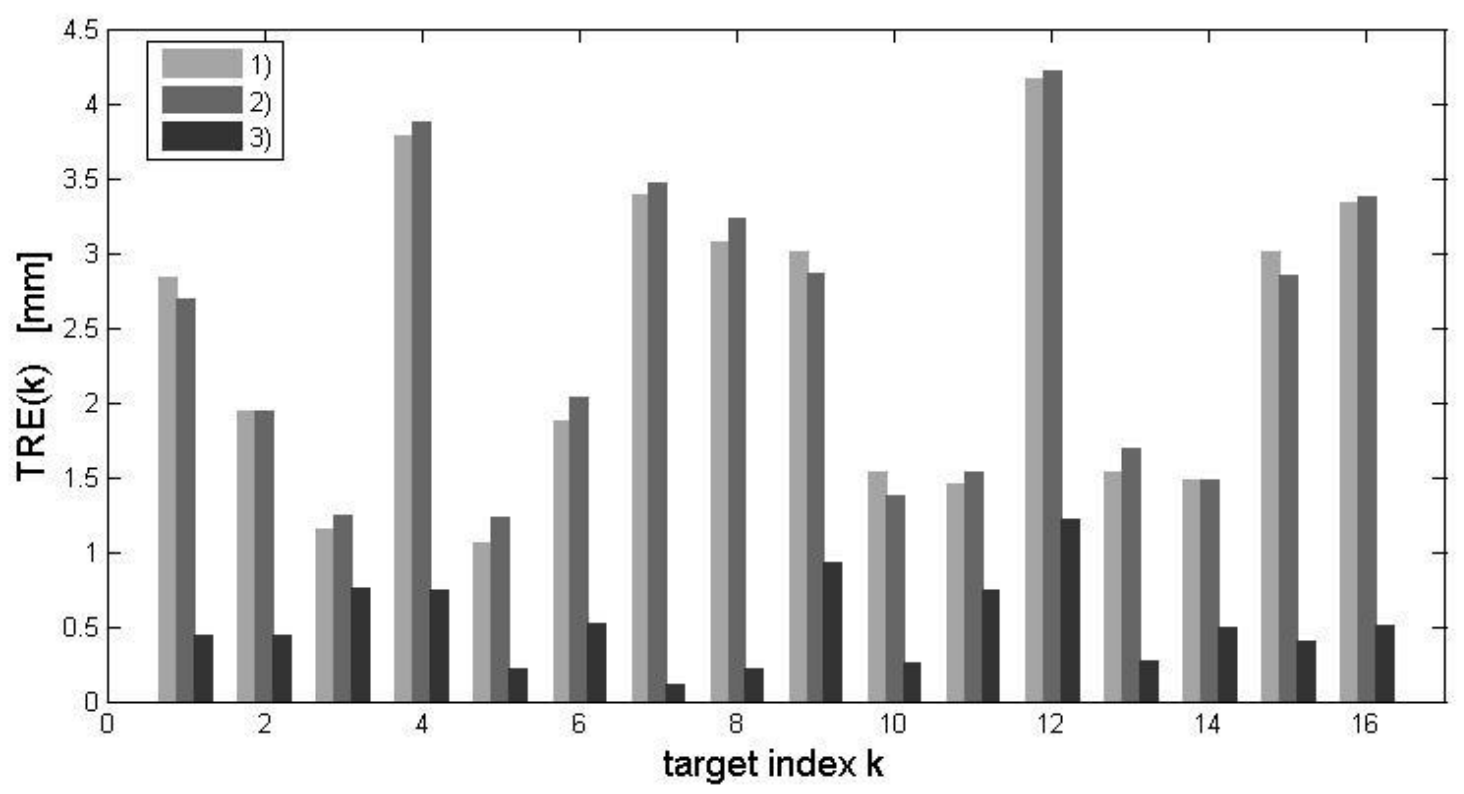

Figure 11. Target registration errors $\operatorname{TRE}(k)$ for data acquired with System A calculated for three post-processing scenarios: 1) uncorrected fiducial and target locations; 2) corrected fiducial location only; 3) corrected fiducial and target locations.

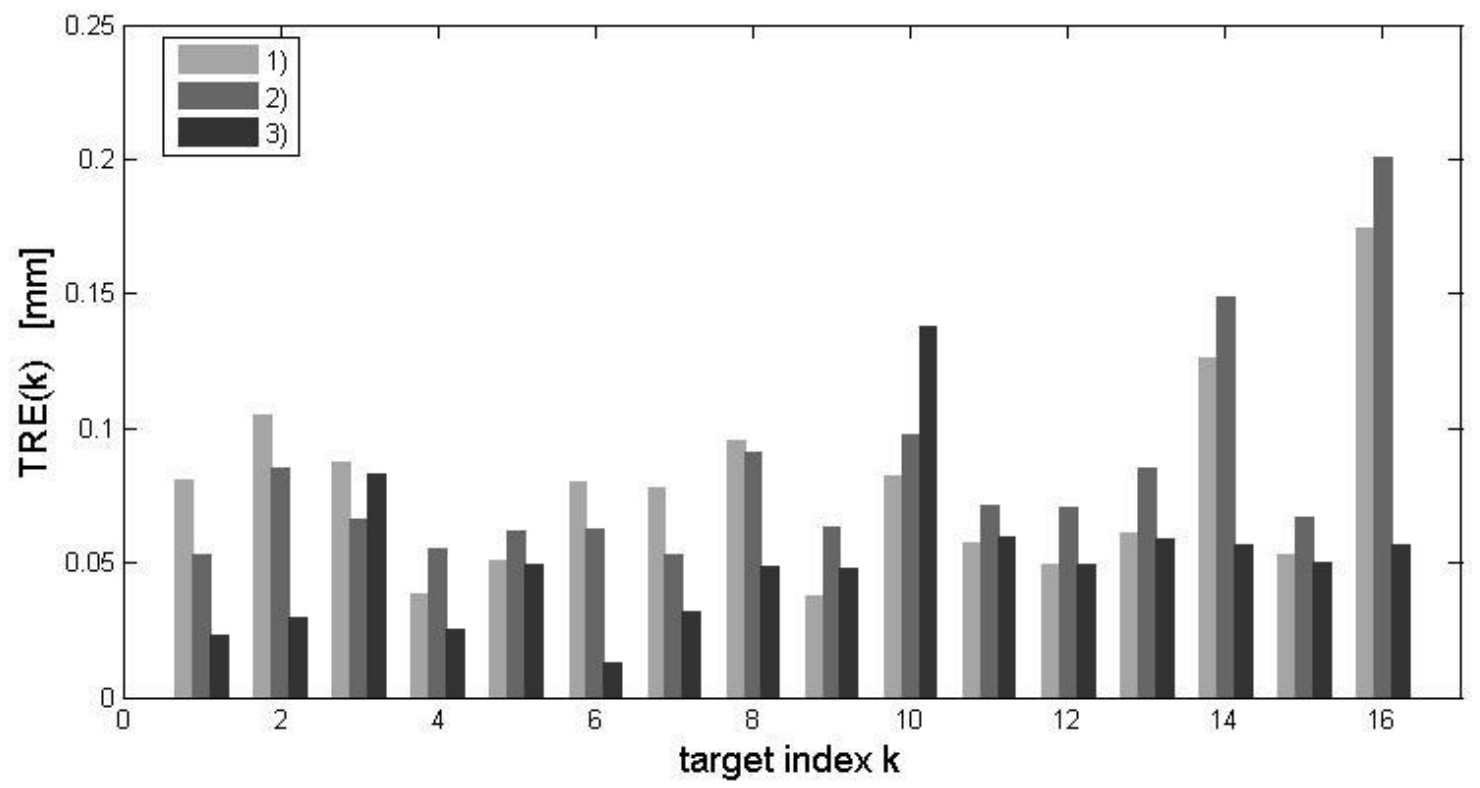

Figure 12. Target registration errors $\operatorname{TRE}(k)$ for data acquired with System B calculated for three post-processing scenarios: 1$)$ uncorrected fiducial and target locations; 2) corrected fiducial locations only; 3) corrected fiducial and target locations. 

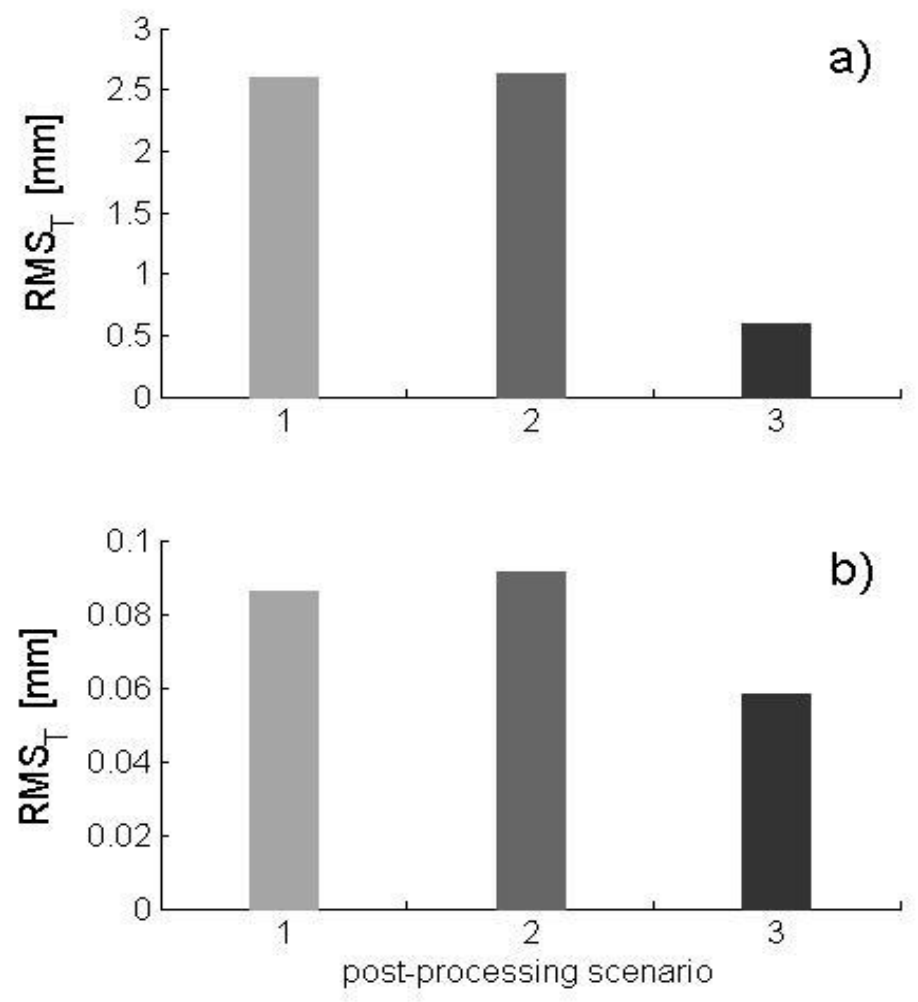

Figure 13. $R M S_{T}$ calculated for the three different post-processing scenarios 1 to 3. a) data acquired with System A; b) data acquired with System B.

\section{DISCUSSION AND FINDINGS}

The histograms of differences $L$ in Figure 3 and Figure 4 show that two iterations of the procedure were sufficient to restore the rigid-body condition for the fiducials in the working frame. The mean of $L$ as well as its spread reduced to almost zero (the range of $L$ values displayed in Figure $3 \mathrm{c}$ and Figure $4 \mathrm{c}$ confirms the numerical convergence of the procedure; physical measurements at the level of $10^{-8} \mathrm{~mm}$ are not achievable by System A, B and LT). Figure 5 and Figure 7 reveal some regular pattern in the spatial distribution of corrections for System A. In particular, the z-component in Figure 5c shows noticeable and similar periodicity for all layers. Note that in Figure 5 and Figure 6, every 25 fiducials constitute a horizontal layer of the $5 \times 5 \times 5$ grid of 
fiducials. In Figure 7, the magnitude of the correction increases with the distance $\overline{\boldsymbol{X}}_{j}$ to the center of the work volume. For the data acquired with System B, the regularity of the observed pattern is weaker and the corrections seem to differ for different layers indicating a more complex bias function.

Plots of differences $g$ in Figure 9 and Figure 10 document how the rigid-body condition is satisfied when both fiducials and target locations are corrected. A large value of $g(k, j)$ calculated using Eq. (23) indicates that the rigid-body condition is violated for the pair of $k$-th target and $j$-th fiducial. Graphs in Figure 9a and Figure 10a are based on the original, uncorrected locations of fiducials and targets, and graphs in Figure 9b and Figure 10b are obtained for the corrected fiducial locations and for target locations modified by the estimated corrections. It is clear that the corrections lead to improvement: rigid-body condition is less violated as is evident from the mean value $\langle g(k)\rangle$ which is closer to zero (compare locations of square markers in Figure 9a, Figure 10a with Figure 9b, Figure 10b, respectively) as well from the reduced difference between the maximum and the minimum value of $g(k, j)$ over all fiducials $j \leq J$. We note that neither the differences $L$ shown in Figure 3 and Figure 4 nor the differences $g$ in Figure 9 and Figure 10 require prior calculation of registration transformation $\{\boldsymbol{R}, \boldsymbol{\tau}\}$. However, knowledge of mean targets locations $\{\overline{\boldsymbol{Y}}\}_{K}$ in the destination frame is needed to calculate $g$.

Since the corrections in the fiducial and target locations lead to better compliance with the rigid-body condition, it is no surprise that the registration transformation $\{\boldsymbol{R}, \boldsymbol{\tau}\}$ based on corrected fiducials and applied to the corrected target in the working frame (via tetrahedral interpolation) yields smaller target error $\operatorname{TRE}(k)$. Substantial reduction in $\operatorname{TRE}(k)$ occurs for all $K=16$ targets for data acquired with System A, as shown in Figure 11, compare scenarios 1 and 3. For some targets (for example: $k=7$ ), the reduced TRE is almost ten times smaller. On average, the corresponding $R M S_{T}$ for all $K$ targets is reduced by a factor of about four, compare scenarios 1 and 3 in Figure 13a. It is crucial to recognize that the corrections in fiducials only without corrections in target location lead to little or no improvement of $R M S_{T}$, see scenario 2 in Figure 11 and Figure 13a. For data acquired with System B, the overall improvement is smaller, compare scenarios 1 and 3 in Figure 
$13 \mathrm{~b} ; R M S_{T}$ is reduced by about $30 \%$ as compared to about $75 \%$ for System A. In fact, for some targets, the error $\operatorname{TRE}(k)$ is larger after the correction is applied when compared with the error for the original, uncorrected fiducials and target, see for example: $k=10$ and 9 for scenarios 1 and 3 in Figure 12. For data acquired with System B, correction in fiducials only without correction in target location lead to worse results, see scenario 2 Figure 12 and Figure 13b.

Based on the results in Figure 11 - Figure 13, two important questions are raised: 1) why is the relative improvement for data acquired with System B smaller than for data acquired with System A when the expectation is that the performance would be similar since the same procedure was used; and 2) what can be done to improve the outcome of the method introduced in this paper? To answer the first question, one must explore the differences between data sets acquired with System A and B. The first most noticeable difference is the degree of compliance with the rigid-body condition: data A violate this condition much more than data B (compare Figure 3a with Figure 4a and Figure 9a with Figure 10a). Thus, one may conclude that, paradoxically, the method works better for worse data, i.e., data which require larger correction. However, since the improvement is based on the ratio of corrected to uncorrected $R M S_{T}$ and not on a comparison of absolute values, this reasoning does not hold. However, we mentioned earlier another difference between data A and B: data from System A has a higher degree of regularity than data from System B. It appears that the dependence of correction on the position, i.e., the function $\boldsymbol{\varepsilon}(\boldsymbol{X})$, for System B may be more complicated and have larger high frequency components than the function $\boldsymbol{\varepsilon}(\boldsymbol{X})$ for System A. While evaluation of corrections $\boldsymbol{\varepsilon}_{j}$ at discretely sampled fiducials $\boldsymbol{X}_{j}$ does not depend on the smoothness of the function $\boldsymbol{\varepsilon}(\boldsymbol{X})$, the performance of the tetrahedral interpolation (or any interpolating scheme) at target $\boldsymbol{T}_{x}$ depends on the sampling density of fiducials $\{\boldsymbol{X}\}_{J}$ in the working volume and the smoothness of the function $\boldsymbol{\varepsilon}(\boldsymbol{X})$. This observation suggests an answer to the second question. We expect that for data acquired with System B the method could yield equally good results as obtained for data A if a larger number $J$ of fiducials were measured in the working and reference 
frames, to account for the high frequency components in the function $\boldsymbol{\varepsilon}(\boldsymbol{X})$. Alternatively, instead of using linear interpolation, a non-linear interpolation (for example: tricubic interpolation [6]) may be better, but these approaches would also require a more dense sampling of fiducials.

It needs to be restated that the restoration of the rigid-body condition can be achieved in various ways, and the set of corrections $\{\boldsymbol{\varepsilon}\}_{J}$ evaluated at fiducials locations $\{\overline{\boldsymbol{X}}\}_{J}$ in the working frame is not unique. However, we want to mention one property of the corrections $\{\boldsymbol{\varepsilon}\}_{J}$ which makes them well defined: namely, the sum of their squares $\sum_{j=1}^{J} \varepsilon_{j}^{2}$ is the smallest from all possible corrections which can restore the rigid-body condition in Eq. (7) (for a given set of fiducials $\{\overline{\boldsymbol{X}}\}_{J}$ and $\{\overline{\boldsymbol{Y}}\}_{J}$ ). We do not have analytical proof for this statement but numerical results strongly support this conjecture. We calculated the registration transformation $\left\{\boldsymbol{R}_{0}, \boldsymbol{\tau}_{0}\right\}$ between the original fiducials $\{\overline{\boldsymbol{X}}\}_{J}$ and the fiducials $\{\widetilde{\boldsymbol{X}}\}_{J}$ corrected by Eq. (15). The resulting transformation was close (within numerical round-off error) to the identity transformation, i.e., $\boldsymbol{\tau}_{0} \approx \mathbf{0}$ and $\boldsymbol{R}_{0} \approx \boldsymbol{I}_{3 \times 3}$. Since the pointbased rigid-body registration minimizes the least squared distance between both sets of fiducials, this means that $\sum_{j=1}^{J} \varepsilon_{j}^{2}$ is the smallest.

Finally, we need to caution against a possible improper implementation of the procedure introduced in this paper. At the beginning of section II, we stated that the noise has to be filtered out from the locations of the fiducials in both the working and destination frames and from targets in the working frame. The method discussed here is based on the mean locations of fiducials and targets. Modern instruments allow users to acquire repeated measurements quickly so the requirement for many repeated measurements should not be a severe limitation. However, due to time or other constraints, one may attempt to use a single, instantaneous measurement of fiducials and target positions instead of the mean of repeated values. This may negate the method which is based on the interpolation of corrections (biases) from four nearby fiducials since any interpolation scheme must be based on time independent, noise free locations of fiducials and targets. Applying 
our method to instantaneous locations of fiducials instead of recommended average locations may be considered only in experimental conditions where noise is few times smaller than observed bias.

\section{FINAL REMARKS}

We introduced a new method to improve point-based, rigid-body registration by restoring the rigid-body condition distorted by bias in measurement or by using fiducials attached to a non-rigid object. Improved registration can be achieved by correcting the locations of fiducials in the working frame and then correcting the target locations by the linearly interpolated corrections from nearby fiducials. The method has been shown to reduce the target registration error in the experimentally obtained datasets. The degree of reduction depends on two factors: the smoothness of the position dependent correction $\boldsymbol{\varepsilon}(\boldsymbol{X})$ and the number of measured fiducials. A smaller number of fiducials is sufficient for correction that slowly varies within the work volume; a larger number is required when the correction is a more complicated, sharply varying function $\boldsymbol{\varepsilon}(\boldsymbol{X})$ within the work volume.

\section{REFERENCES}

[1] Moghari MH, Abolmaesumi P (2010) Understanding the Effect of Bias in Fiducial Localization Error on Point-Based Rigid-Body Registration. IEEE Trans Medical Imaging 29(10):1730-1738.

[2] Franaszek M , Cheok GS (2017) Selection of fiducial locations and performance metrics for point-based rigid-body registration. Precision Engineering 47:362-374.

[3] Bourke P (1999) Interpolation methods. Available at http://paulbourke.net/miscellaneous/interpolation/. Accessed 08/31/2016,

[4] Hemingway P (2002) n-Simplex Interpolation. Available at http://www.hpl.hp.com/techreports/2002/HPL-2002-320.pdf. Accessed 08/30/2016,

[5] Kasson JM, Plouffe W, Nin SI (1993) Tetrahedral interpolation technique for color space conversion. Proc SPIE 1909 Device-Independent Color Imaging and Imaging Systems Intergration, (SPIE), p 127. https://doi.org/10.1117/12.149035

[6] Lekien F , Marsden J (2005) Tricubic interpolation in three dimensions. Int J Numerical Methods in Engineering 63:455471. https://doi.org/10.1002/nme.1296 